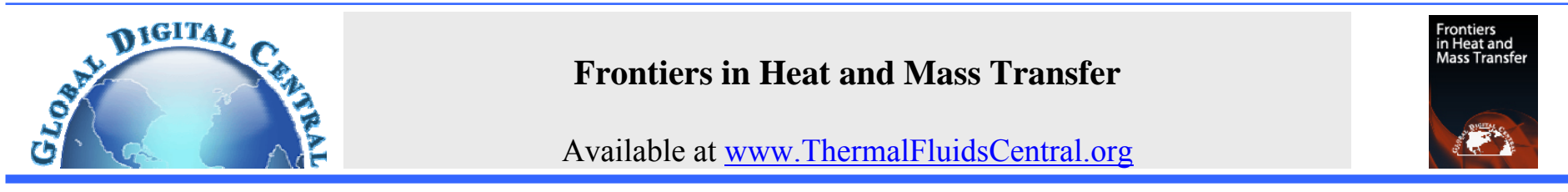

\title{
HEAT AND MASS TRANSFER AND ENTROPY GENERATION INSIDE 3D TRAPEZOIDAL SOLAR DISTILLER
}

\author{
Walid Aich ${ }^{\mathrm{a}, \mathrm{c}}$, Lioua Kolsi ${ }^{\mathrm{a}, \mathrm{d}^{*}}$, Abdelkarim Aydie,f ${ }^{\mathrm{e}}$, Abdullah A.A.A Al-Rashed ${ }^{\mathrm{b}}$, Noureddine Ait Messaoudene, \\ Mohamed Naceur Borjini ${ }^{\mathrm{d}}$ \\ ${ }^{a}$ Mechanical Engineering Department, College of Engineering, Haïl University, Haïl City, Saudi Arabia \\ ${ }^{b}$ Dept. of Automotive and Marine Engineering Technology, College of Technological Studies, The Public Authority for Applied Education and \\ Training, Kuwait \\ 'Unité de Recherche Matériaux, Energie et Energies Renouvelables, Faculté des Sciences de Gafsa, Tunisie \\ ${ }^{d}$ Unité de Recherche de Métrologie et des Systèmes Energétiques, Ecole Nationale d'Ingénieurs, 5000 Monastir, University of Monastir, Tunisia \\ ${ }^{e}$ Department of Chemical and Materials Engineering, College of Engineering, Northern Border University, P.O. Box 1321, Arar, Saudi Arabia \\ fLaboratoire Matériaux Molécules et Applications-IPEST La Marsa, Tunisia
}

\begin{abstract}
Numerical study of double-diffusive natural convection flow and entropy generation in 3D trapezoidal solar distiller was performed using computational fluid dynamics (CFD). In this research the flow, provoked by the interaction of chemical species diffusions and the thermal energy, is assumed to be laminar. Using potential vector-vorticity formulation in its three-dimensional form, the governing equations are formulated and solved by the numerical methodology based on the finite volume method. The main objective is to analyze the effects of buoyancy ratio for opposed temperature and concentration gradients and to focus the attention on three-dimensional aspects and generated entropy. The occurring heat and mass transfer are depicted by the dynamic and temperature fields of the flow inside the trapezoidal cavity. It has been found that the flow structure and the heat and mass transfer are sensitive to the value of buoyancy ratio.
\end{abstract}

Keywords: Three-dimensional flow, Entropy generation, Solar energy, 3-D CFD, Double diffusive natural convection, Entropy generation, Trapezoidal Solar distiller.

\section{INTRODUCTION}

The accessibility to drinking water is one of the main problems for human being in arid remote areas all over the world. In addition, there as well are many coastal locations where sea water is abundant but potable water is not available. Solar stills, where brackish or sea water is evaporated and is then condensed as pure water, can solve part of this problem. In such processes conditions are especially favorable for double-diffusive natural convection flow to occur. This convection is generated by buoyancy due to simultaneous temperature and concentration gradients. A sizable amount of research interests has been addressed on this subject (Mirabedin, 2016; Alam et al. 2016; Bouabdallah et al. 2016; Bennacer et al. 2001; Béghein et al. 1992; Han et al. 1992; Bennacer et al. 1996; Wee et al. 1989; Chen et al. 2011; Gobin et al. 1996; Ghachem et al. 2012; Tofaneli et al. 2009; Costa, 2004). The outcome shows the occurrence of an oscillatory flow, provoked by the equilibrium between thermal and compositional forces, for a buoyancy number near the unity.

Nishumira et al. (1998) performed a careful analysis of the mechanism of such oscillatory flow. The majority of numerical investigations is restricted to the two-dimensional geometry and only very limited work has been done on 3D double diffusive natural convection. Opposing gradients thermosolutal natural convection in a cubic cavity was carried out by Sezai et al. (2000). They mentioned that the $3 \mathrm{D}$ character is dominant for a certain range of parameters. Abidi et al. (2008) studied the same configuration with heat and mass diffusive horizontal walls. The results show that there is a great difference between the cases of the compositionally dominated flow and thermally dominated one. They also mentioned that for solutally dominated flow, an "inverse transition" from a multicellular to a unicellular pattern occurs. Meftah et al. (2009) conducted a study of the combined radiation double diffusive natural convection. An oblique stratification on the temperature and concentration fields due to the influence of radiation was observed. In a second study, Abidi et al. (2011) exanimated the thermal radiation effect on three-dimensional double diffusive natural convection. They pointed out that the flow structure is extremely sensitive to the variations of the conduction-radiation parameter and optical thickness. The double diffusive convection with Soret effect inside a square porous enclosure heated and salted from below was examined by Khadiri et al. (2010). It was observed that both, transition to oscillatory regime and heat transfer are significantly affected by the Soret parameter.

Ben Niche et al. (2016) have numerically investigated the transient double diffusive natural convection in a square cavity. The results showed that both heat and mass transfer increased in the presence of Dufour coefficient, and the Soret coefficient had great effect on the flow structure. The double diffusive convection flow at high Rayleigh number was studied by Li et al. (2008). They concluded that threedimensional modeling should be carried out so as to have better

*Corresponding author. Email: lioua_enim@yahoo.fr 
agreement with experimental data. Besides, the stratified system is found to be sensitive to the buoyancy ratio. Sampathkumar et al. (2010) presented a detailed literature survey on the subject of modeling of active solar distillation procedures. The most used technology for the solar desalination of brackish water was the capillary film distiller (Bouchekima et al. (1999, 2000, 2003)).

Moreover, only limited attention has been paid to the study of three-dimensional transverse flow which is primordial when dealing with the enhancement of heat and mass transfers. The main objective of this research is to numerically investigate the double diffusive natural convection in a three-dimensional trapezoidal solar distiller, for a buoyancy ratio ranging from -10 to 10 .

\section{PHYSICAL PROBLEM AND GOVERNING EQUATIONS}

The considered configuration (Fig. 1) represents a three dimensional trapezoidal solar distiller cavity of equal side's length $\mathrm{W}$. The bottom and the sloping top wall are maintained to different and uniform temperatures and concentrations while all other walls are assumed to be insulated. Due to solar radiation, the hot wall warms up which leads to the evaporation of the water film; subsequently, this vapor after being mixed with the air condenses in contact of the cold wall. The enclosure is assumed to be filled by an incompressible fluid and the flow follows the Boussinesq approximation.

Governing equations which are respectively equations of continuity, momentum, energy and species diffusion are written as:

$$
\begin{aligned}
& \nabla \cdot \vec{V}^{\prime}=0 \\
& \frac{\partial \vec{V}^{\prime}}{\partial t^{\prime}}+\left(\overrightarrow{V^{\prime}} \cdot \vec{\nabla}\right) \vec{V}^{\prime}=-\frac{1}{\rho} \vec{\nabla} P^{\prime}+v \Delta \vec{V}^{\prime}+\beta_{t}\left(T^{\prime}-T_{0}\right) \vec{g}+\beta_{C}\left(C^{\prime}-C_{0}\right) \vec{g} \\
& \frac{\partial T^{\prime}}{\partial t^{\prime}}+\vec{V}^{\prime} . \nabla T^{\prime}=\alpha \nabla^{2} T^{\prime} \\
& \frac{\partial C^{\prime}}{\partial t^{\prime}}+\vec{V}^{\prime} \cdot \nabla C^{\prime}=D \nabla^{2} C^{\prime}
\end{aligned}
$$

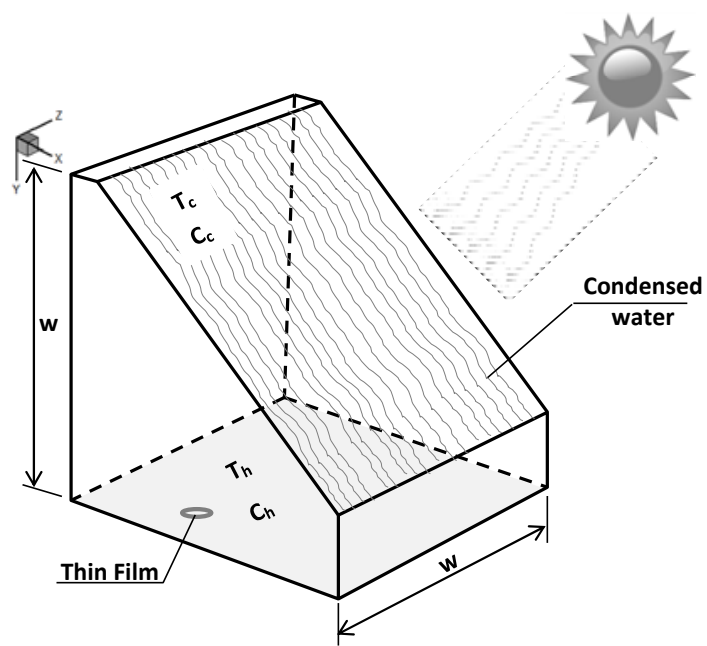

Fig. 1 Geometric configuration

As numerical method we had recourse to the potential vectorvorticity formalism $(\vec{\psi}-\vec{\omega})$ which allows eliminating the pressure gradient. To remove of this term, the rotational is applied to the equation of momentum. The potential vector and the vorticity are respectively described by the two following relations:

$\vec{\omega}^{\prime}=\vec{\nabla} \times \vec{V}^{\prime}$ and $\vec{V}^{\prime}=\vec{\nabla} \times \vec{\psi}^{\prime}$

In the equations $(1,2,3$ and 4$)$, time $t^{\prime}$, velocity $\vec{V}^{\prime}$, the stream function $\vec{\psi}^{\prime}$, the vorticity $\vec{\omega}^{\prime}$, are put respectively in their dimensionless forms by $W^{2} / \alpha, \alpha / W, \alpha$ and $W^{2} / \alpha$ : and the dimensionless temperature and concentration are respectively defined by: $T=\left(T^{\prime}-T_{C}{ }^{\prime}\right) /\left(T_{h}{ }^{\prime}-T_{C}{ }^{\prime}\right)$ and $C=\left(C^{\prime}-C_{l}{ }^{\prime}\right) /\left(C_{h}{ }^{\prime}-C_{l}{ }^{\prime}\right)$.

After applying the $(\vec{\psi}-\vec{\omega})$ formalism and using the dimensionless form, the system of equations governing the phenomenon becomes as:

$$
\begin{gathered}
-\vec{\omega}=\nabla^{2} \vec{\psi} \\
\frac{\partial \vec{\omega}}{\partial t}+(\vec{V} \cdot \nabla) \vec{\omega}-(\vec{\omega} \cdot \nabla) \vec{V}=\Delta \vec{\omega}+\operatorname{Ra} \cdot \operatorname{Pr} \cdot\left[\frac{\partial T}{\partial z}-N \frac{\partial C}{\partial z} ; 0 ;-\frac{\partial T}{\partial x}+N \frac{\partial C}{\partial x}\right] \\
\frac{\partial T}{\partial t}+\vec{V} \cdot \nabla T=\nabla^{2} T \\
\frac{\partial C}{\partial t}+\vec{V} \cdot \nabla C=\frac{1}{L e} \nabla^{2} C \\
\text { With } \operatorname{Pr}=v / \alpha, R a=\frac{g \cdot \beta_{t} \cdot W^{3} \cdot\left(T_{h}^{\prime}-T_{c}^{\prime}\right)}{\alpha \cdot v}, N=\frac{\beta_{c} \cdot\left(C_{h}^{\prime}-C_{l}^{\prime}\right)}{\beta_{t} \cdot\left(T_{h}^{\prime}-T_{c}^{\prime}\right)}, \\
L e=\frac{\alpha}{D}=\frac{S c}{\operatorname{Pr}}
\end{gathered}
$$

The boundary conditions are given as:

\section{- $\quad$ Temperature}

$T=1 \quad$ at $\quad y=0, \quad T=0$ at inclined wall; $\frac{\partial T}{\partial n}=0$ on other walls (adiabatic)

\section{- Concentration}

$C=1 \quad$ at $y=0, \quad C=0$ at inclined wall; $\frac{\partial C}{\partial n}=0$ on other walls (impermeable).

$$
\begin{gathered}
\bullet \quad \text { Vorticity } \\
\omega_{x}=0, \omega_{y}=-\frac{\partial V_{z}}{\partial x}, \omega_{z}=\frac{\partial V_{y}}{\partial x} \text { at } x=0 \text { and } 1 ; \omega_{x}=\frac{\partial V_{z}}{\partial y}, \omega_{y}=0, \\
\omega_{z}=-\frac{\partial V_{x}}{\partial y} \text { at } y=0 \text { and } 1 ; \omega_{x}=-\frac{\partial V_{y}}{\partial z}, \omega_{y}=\frac{\partial V_{x}}{\partial z}, \omega_{z}=0 \text { at } z=0
\end{gathered}
$$

and 1

\section{- Potential vector}

$\frac{\partial \psi_{x}}{\partial x}=\psi_{y}=\psi_{z}=0$ at $x=0$ and $1 ; \psi_{x}=\frac{\partial \psi_{y}}{\partial y}=\psi_{z}=0$ at $y=0$ and 1 ;

$\psi_{x}=\psi_{y}=\frac{\partial \psi_{z}}{\partial z}=0$ at $z=0$ and 1

$$
\begin{gathered}
\bullet \quad \text { velocity } \\
V_{x}=V_{y}=V_{z}=0 \text { on all walls }
\end{gathered}
$$

The local Nusselt and Sherwood numbers at the hot wall have the expressions as:

$$
N u=\left.\frac{\partial T}{\partial y}\right|_{y=0} \quad \text { and } \quad S h=\left.\frac{\partial C}{\partial y}\right|_{y=0}
$$

The average values of the numbers of Nusselt and Sherwood, on the isothermal walls are expressed by:

$$
N u_{a v}=\int_{0}^{1} \int_{0}^{1} N u . \partial y . \partial z \quad \text { and } \quad S h_{a v}=\int_{0}^{1} \int_{0}^{1} S h . \partial y . \partial z
$$

In a $3 \mathrm{D}$ flow with single diffusing specie of concentration (C), the local entropy generation rate can be written as (Ghachem et al. (2012)). 


$$
\begin{aligned}
& S_{\text {gen }}^{\prime}=\left\{\frac{k}{T_{0}^{2}}\left[\left(\frac{\partial T^{\prime}}{\partial x^{\prime}}\right)^{2}+\left(\frac{\partial T^{\prime}}{\partial y^{\prime}}\right)^{2}+\left(\frac{\partial T^{\prime}}{\partial z^{\prime}}\right)^{2}\right]\right\} \\
& +\frac{\mu}{T_{0}}\left\{2\left(\frac{\partial V_{x}^{\prime}}{\partial x^{\prime}}\right)^{2}+\left(\frac{\partial V_{y}^{\prime}}{\partial y^{\prime}}\right)^{2}+\left(\frac{\partial V_{z}^{\prime}}{\partial z^{\prime}}\right)^{2}\right] \\
& \left.+\left(\frac{\partial V_{y}^{\prime}}{\partial x^{\prime}}+\frac{\partial V_{x}^{\prime}}{\partial y^{\prime}}\right)^{2}+\left(\frac{\partial V_{z}^{\prime}}{\partial y^{\prime}}+\frac{\partial V_{y}^{\prime}}{\partial z^{\prime}}\right)^{2}+\left(\frac{\partial V_{x}^{\prime}}{\partial z^{\prime}}+\frac{\partial V_{z}^{\prime}}{\partial x^{\prime}}\right)^{2}\right\} \\
& +\left\{\begin{array}{l}
\frac{R D}{C_{0}}\left[\left(\frac{\partial C^{\prime}}{\partial x^{\prime}}\right)^{2}+\left(\frac{\partial C^{\prime}}{\partial y^{\prime}}\right)^{2}+\left(\frac{\partial C^{\prime}}{\partial z^{\prime}}\right)^{2}\right] \\
+\frac{R D}{T_{0}}\left[\left(\frac{\partial T^{\prime}}{\partial x^{\prime}}\right)\left(\frac{\partial C^{\prime}}{\partial x^{\prime}}\right)+\left(\frac{\partial T^{\prime}}{\partial y^{\prime}}\right)\left(\frac{\partial C^{\prime}}{\partial y^{\prime}}\right)+\left(\frac{\partial T^{\prime}}{\partial z^{\prime}}\right)\left(\frac{\partial C^{\prime}}{\partial z^{\prime}}\right)\right]
\end{array}\right\}
\end{aligned}
$$

Where $\mathrm{C}_{0}$ and $\mathrm{T}_{0}$ are respectively the reference concentration and temperature,

In its dimensionless form the local generated entropy is written as:

$$
\begin{aligned}
& N_{S}=\left[\left(\frac{\partial T}{\partial x}\right)^{2}+\left(\frac{\partial T}{\partial y}\right)^{2}+\left(\frac{\partial T}{\partial z}\right)^{2}\right]+ \\
& \left.2\left(\frac{\partial V_{x}}{\partial x}\right)^{2}+\left(\frac{\partial V_{y}}{\partial y}\right)^{2}+\left(\frac{\partial V_{z}}{\partial z}\right)^{2}\right] \\
& \varphi_{1} \cdot\left\{\begin{array}{l}
{\left[\left(\frac{\partial V_{y}}{\partial x}+\frac{\partial V_{x}}{\partial y}\right)^{2}+\left(\frac{\partial V_{z}}{\partial y}+\frac{\partial V_{y}}{\partial z}\right)^{2}+\left(\frac{\partial V_{x}}{\partial z}+\frac{\partial V_{z}}{\partial x}\right)^{2}\right]} \\
+
\end{array}\right\} \\
& +\left\{\begin{array}{l}
\varphi_{2}\left[\left(\frac{\partial C}{\partial x}\right)^{2}+\left(\frac{\partial C}{\partial y}\right)^{2}+\left(\frac{\partial C}{\partial z}\right)^{2}\right]+ \\
\varphi_{3}\left[\left(\frac{\partial T}{\partial x}\right)\left(\frac{\partial C}{\partial x}\right)+\left(\frac{\partial T}{\partial y}\right)\left(\frac{\partial C}{\partial y}\right)+\left(\frac{\partial T}{\partial z}\right)\left(\frac{\partial C}{\partial z}\right)\right]
\end{array}\right\}
\end{aligned}
$$

The first term of NS represents the thermal irreversibility $\left(N_{S-t h}\right)$, the second term represents the viscous irreversibility $\left(\mathrm{N}_{\mathrm{S}-\mathrm{fr}}\right)$ and the third term represents the diffusive irreversibility $\left(N_{S-d i f}\right)$. $N_{S}$ gives a good idea on the distribution and the profile of the local dimensionless entropy generation. The total dimensionless generated entropy is written as follows:

$$
S_{\text {tot }}=\int_{v} N_{s} d v=\frac{1}{v} \int_{v}\left(N_{s-t h}+N_{s-f r}+N_{s-d i f}\right) d v=S_{t h}+S_{f r}+S_{d i f}
$$

Bejan number $(\mathrm{Be})$ is the ratio of heat and mass transfer irreversibility to the total generated entropy as:

$$
B e=\frac{S_{t h}+S_{d i f}}{S_{t h}+S_{f r}+S_{d i f}}
$$

Dimensionless irreversibilities distribution ratios $\left(\varphi_{1}, \varphi_{2}\right.$ and $\varphi_{3}$ ), are given by:

$$
\varphi_{1}=\frac{\mu \alpha^{2} T_{0}}{L^{2} k \Delta T^{2}} ; \varphi_{2}=\frac{R D T_{0}}{k C_{0}}\left[\frac{\Delta C^{\prime}}{\Delta T^{\prime}}\right] ; \varphi_{3}=\frac{R D}{k}\left[\frac{\Delta C^{\prime}}{\Delta T^{\prime}}\right]
$$

For $\mathrm{N}=0$, there is no mass diffusion and we assume that thermal and species diffusions are opposed. For $0<\mathrm{N}<1$ the flow is thermal dominated and for $\mathrm{N}>1$ the flow is Compositional dominated.

The mathematical model described above is written by a FORTRAN program. The control volume method is used to discretize equations $(7,8,9$ and 14). The central-difference scheme is used for treating convective terms while the fully implicit procedure is used to discretize the temporal derivatives. The grids are considered uniform in all directions with clustering nodes on boundaries. The successive relaxation iteration scheme is used to accelerate the convergence.

To handle the irregularly shaped computational domain the blocked-off method was used. In this technique, the whole region is divided into two active and inactive (blocked-off regions) parts (Fig. 2). Thus the inclined surface is approximated by a series of small cubes. Using the blocked-off technique, known values must be established in inactive control volumes; in our case all velocity components are equal to zero and temperature is constant. The control volumes, which are inside the active region, are designated as (1) and otherwise they are $(0)$. The time step $\left(10^{-4}\right)$ and spatial mesh $\left(81^{\times} 81 \times 81\right)$ are utilized to carry out all the numerical tests. The solution is considered acceptable when the following convergence criterion is satisfied for each step of time as:

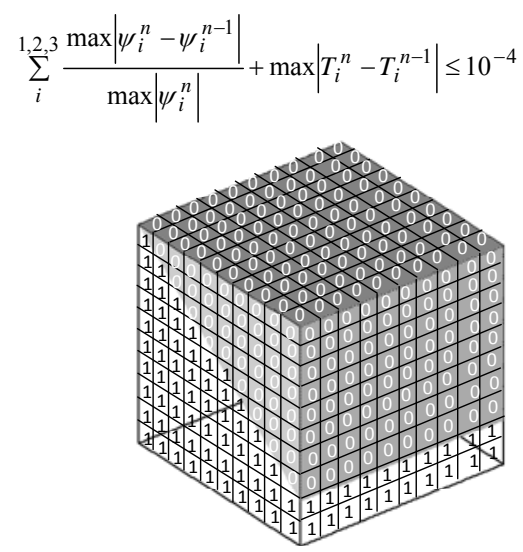

Fig. 2 Blocked-off region in a regular grid

\section{VALIDATION}

The results of this study were compared to those of Sezai et al. (2000), Fig. 3. The carried comparison indicates a perfect agreement with a difference less than $1 \%$ for all values of $\mathrm{N}$.

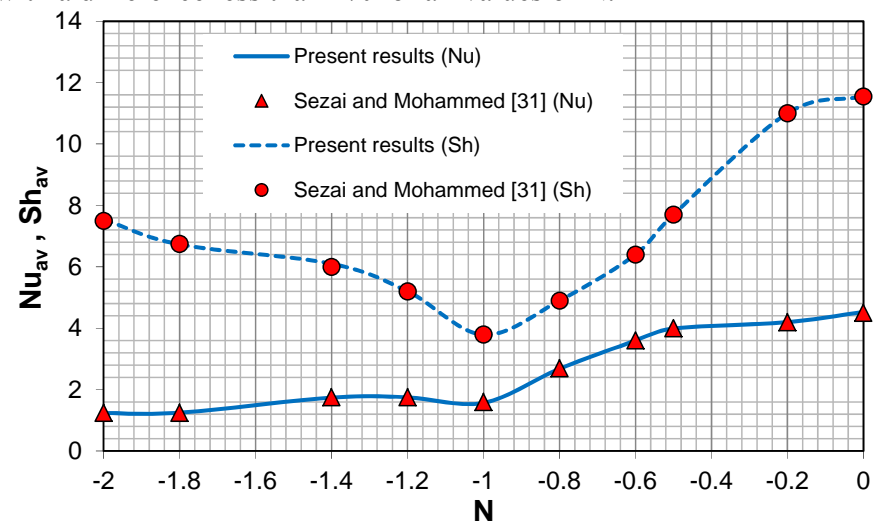

Fig. 3 Comparison between the present results and those of Sezai et al. (2000) $\left(\mathrm{Ra}=10^{5}, \mathrm{Pr}=10\right.$ and $\left.\mathrm{Le}=10\right)$

\section{RESULTS AND DISCUSSIONS}

Numerical results are presented for $\operatorname{Pr}=0.7$ and $\mathrm{Le}=0.85(\mathrm{Sh}=0.6)$ which cover water vapor diffusion into air. The Rayleigh number is varies from $10^{3}$ to $10^{5}$, and the buoyancy ratio ranges from $\mathrm{N}=-10$ to 10. The dimensionless irreversibilities distribution ratios $\left(\varphi_{1}, \varphi_{2}\right.$ and $\varphi_{3}$ ), are fixed respectively at $10^{-4}, 0.5$ and $10^{-2}$ (Ghachem et al. (2012)).

Particle trajectories for different buoyancy ratio, for $\mathrm{Ra}=10^{5}$, are shown in Fig.4. For lower values of buoyancy ratio $(\mathrm{N}=-10$ and $\mathrm{N}=-$ 2 ), the flow is thermal dominated characterized by one central vortex which turns in the anticlockwise direction. For $\mathrm{N}=-1$, the intensity of convection increases and causes secondary vortex to develop on the lower right corner which turns in the clockwise direction. By increasing the buoyancy ratio, we notice that the flow becomes chaotic for $\mathrm{N}=-0.9$ 
and then for $\mathrm{N} \geq-0.5$, the secondary vortex becomes bigger and invades the central part by pushing the primary one until it disappears.

In fig.5, the projection of velocity vector in the XY-plan is depicted. For $\mathrm{N}<-1$ the flow is thermally dominated with only one anticlockwise rotating thermal vortex. Regarding $\mathrm{N}=-1$, it is noticed that a great compositional vortex appears and grows in in size by increasing the buoyancy ratio. Starting from $\mathrm{N}=-0.5$, we note the reappearance of the single vortex structure but with inversed flow rotation caused by the compositional domination and the increase of the $\mathrm{N}$ beyond this value has no effect on the flow structure. it has become obvious, therefore, that the desirable flow pattern would be one whereby the inclined surface was associated with a single cell, which would rotate in a sense that could drive the condensed vapor toward the right lower end of the inclined glass cover (clockwise rotation).

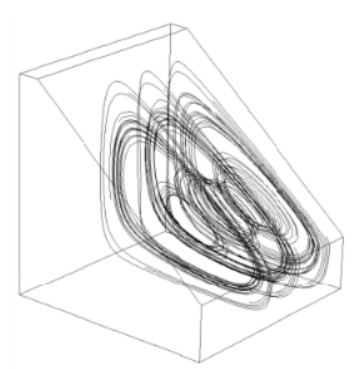

$$
\mathbf{N}=\mathbf{- 1 0}
$$

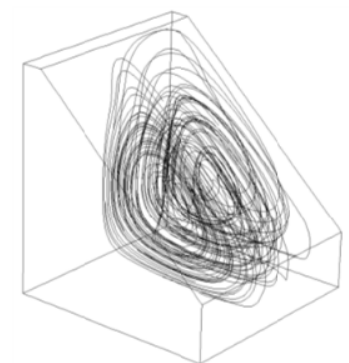

$\mathbf{N}=\mathbf{0}$

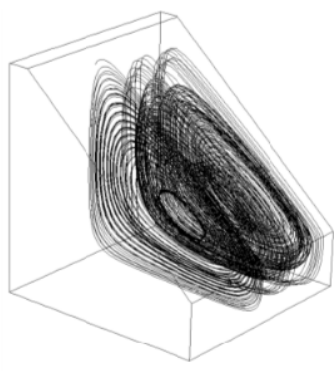

$\mathbf{N}=-\mathbf{2}$

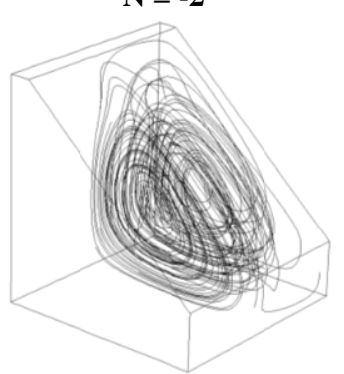

$\mathrm{N}=\mathbf{0 . 5}$
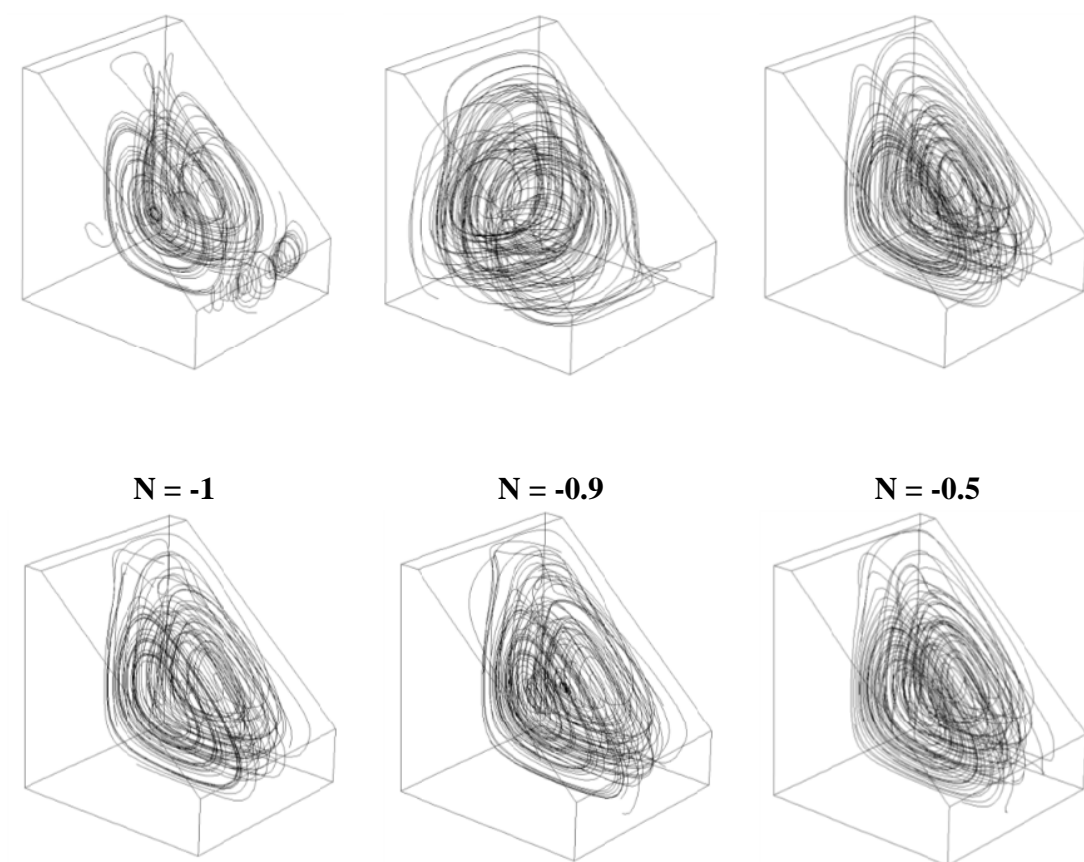

$\mathbf{N}=\mathbf{2}$

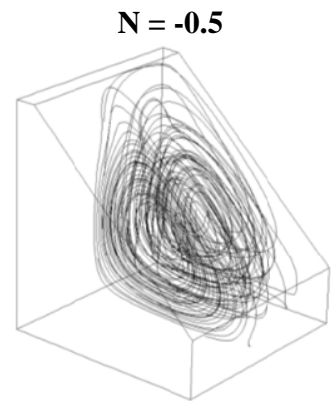

$\mathbf{N}=\mathbf{1 0}$

Fig. 4 Some particles trajectories for different $\mathrm{N} ; \mathrm{Ra}=10^{5}$

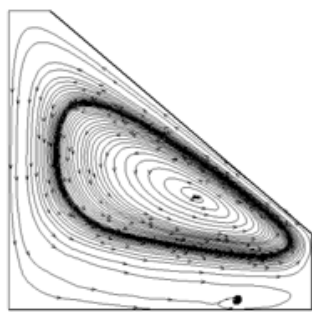

$\mathbf{N}=\mathbf{- 1 0}$

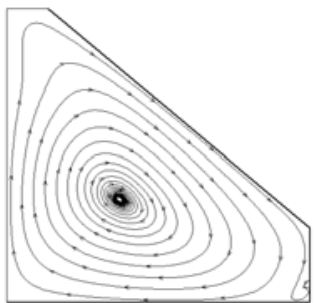

$\mathbf{N}=\mathbf{0}$

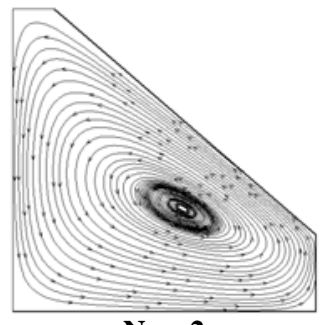

$\mathbf{N}=-2$

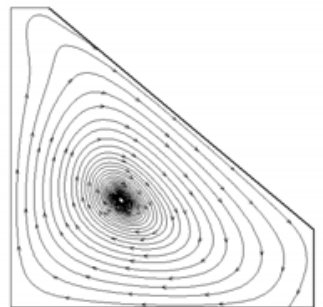

$\mathbf{N}=\mathbf{0 . 5}$

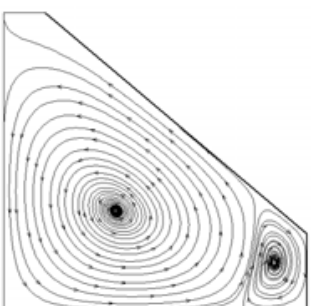

$\mathbf{N}=\mathbf{- 1}$

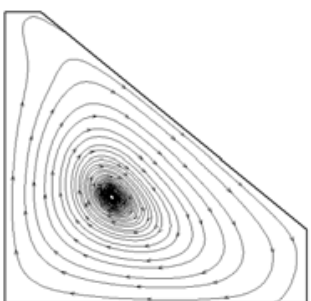

$\mathbf{N}=\mathbf{1}$

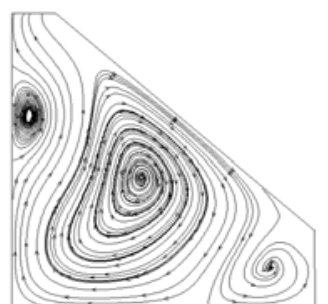

$\mathbf{N}=\mathbf{- 0 . 9}$

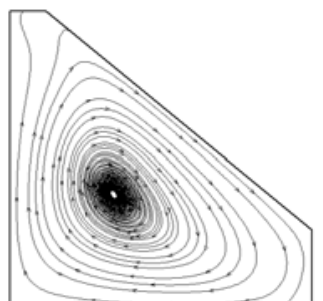

$\mathbf{N}=\mathbf{2}$

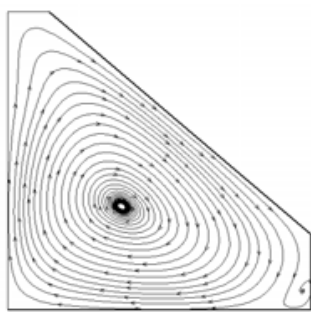

$\mathrm{N}=\mathbf{- 0 . 5}$

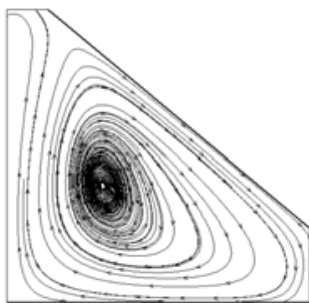

$\mathbf{N}=\mathbf{1 0}$

Fig. 5 Projection of the velocity vector in the central plan (XY-plan) for different $\mathrm{N} ; \mathrm{Ra}=10^{5}$ 

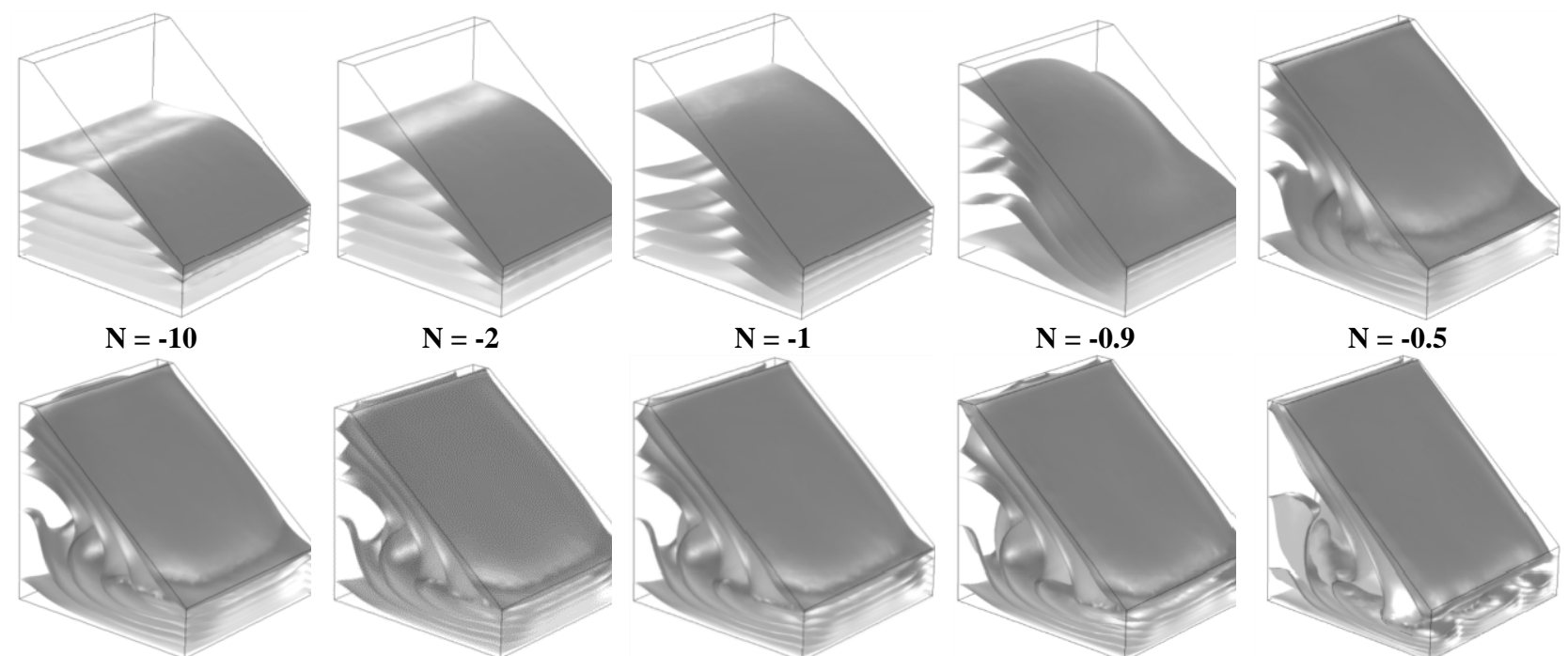

$\mathrm{N}=0.5$

$\mathbf{N}=\mathbf{1}$

$\mathbf{N}=\mathbf{2}$

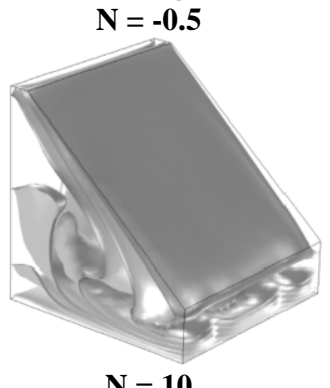

Fig. 6 Iso-surfaces of temperature for different $\mathrm{N}$; $\mathrm{Ra}=10^{5}$
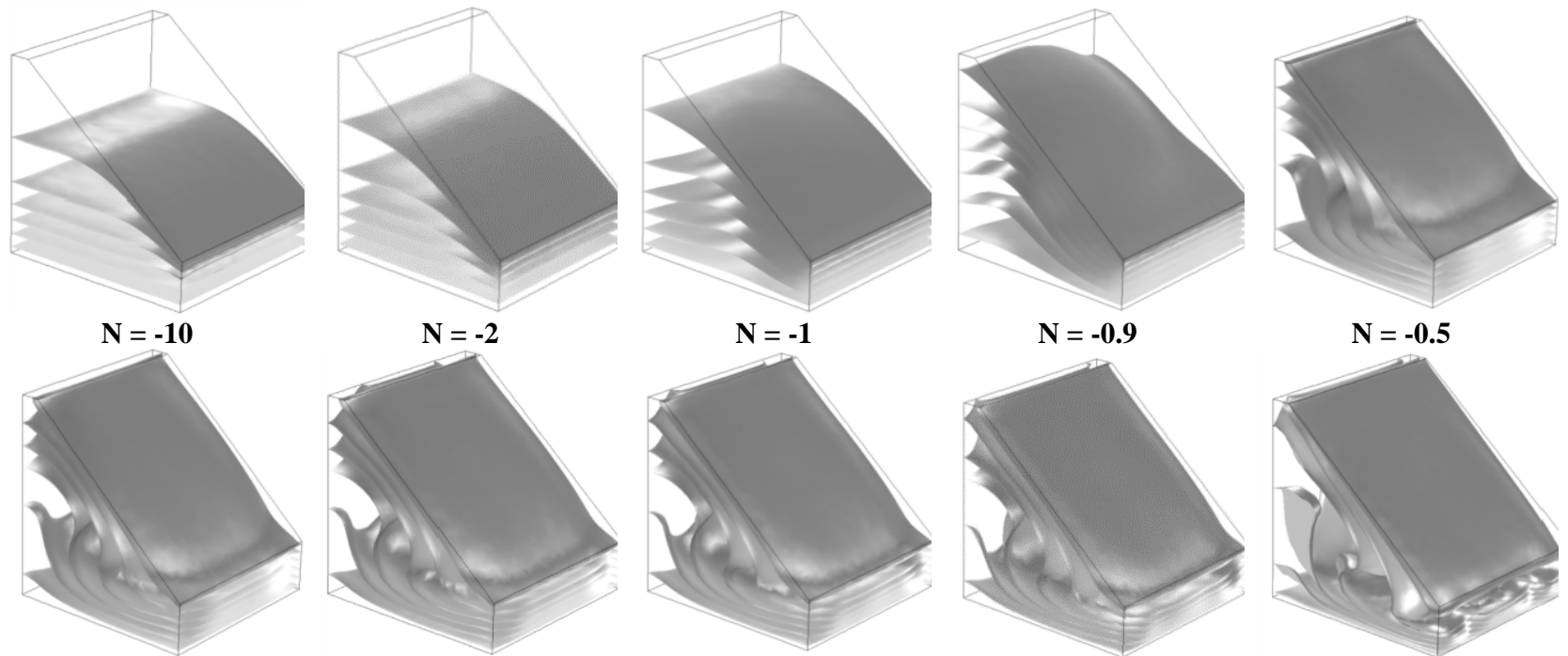

$\mathbf{N}=\mathbf{0}$

$\mathrm{N}=0.5$

$\mathbf{N}=\mathbf{1}$

$\mathbf{N}=\mathbf{2}$

$\mathbf{N}=\mathbf{1 0}$

Fig. 7 Iso-surfaces of concentration for different $\mathrm{N}$; $\mathrm{Ra}=10^{5}$

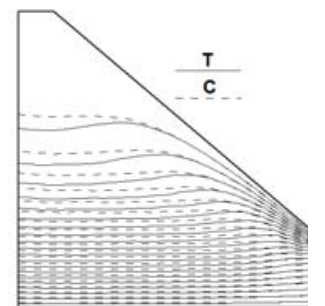

$\mathbf{N}=\mathbf{- 1 0}$

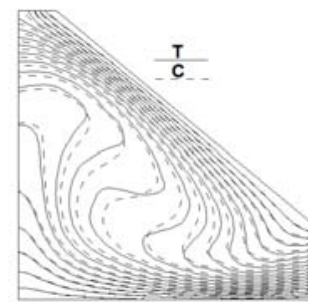

$\mathbf{N}=\mathbf{0}$

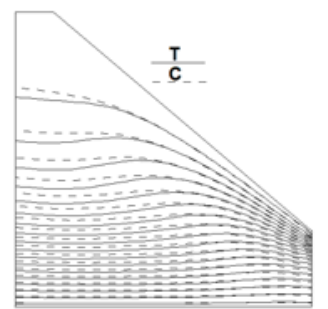

$\mathbf{N}=-\mathbf{2}$

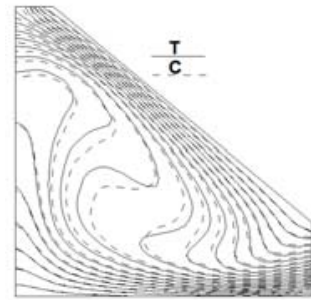

$\mathrm{N}=\mathbf{0 . 5}$

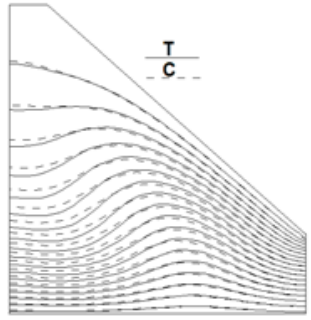

$\mathbf{N}=\mathbf{- 1}$

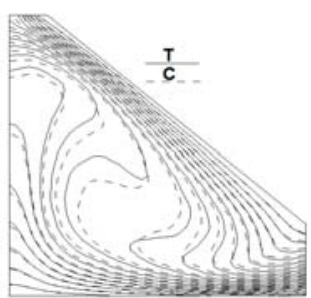

$\mathbf{N}=\mathbf{1}$

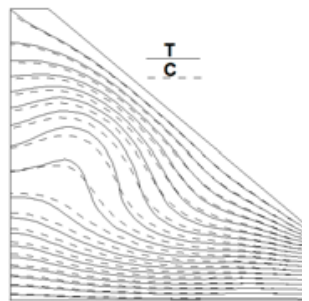

$\mathbf{N}=\mathbf{- 0 . 9}$

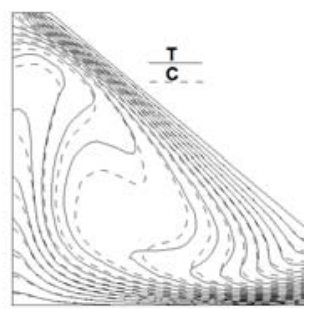

$\mathbf{N}=\mathbf{2}$

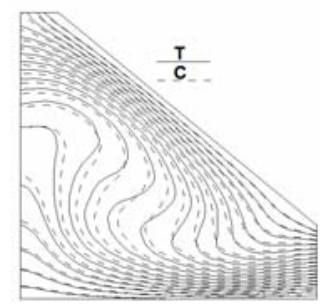

$\mathbf{N}=\mathbf{- 0 . 5}$

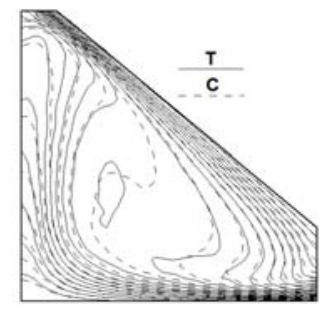

$\mathbf{N}=\mathbf{1 0}$

Fig. 8 Iso-temperatures and iso-concentrations in the XY-plan for different $\mathrm{N}$. 

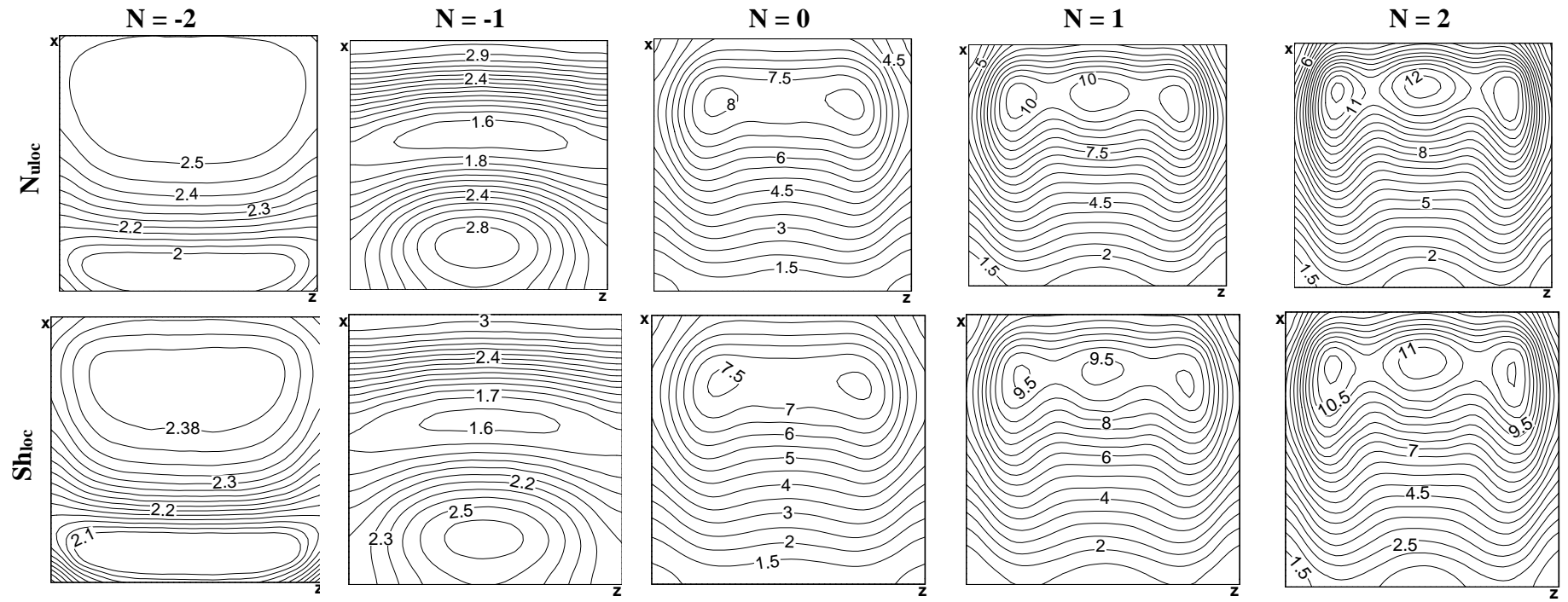

Fig. 9 Local Nusselt number (Top) and local Sherwood number (Bottom) as a function of $\mathrm{N} ; \mathrm{Ra}=10^{5}$
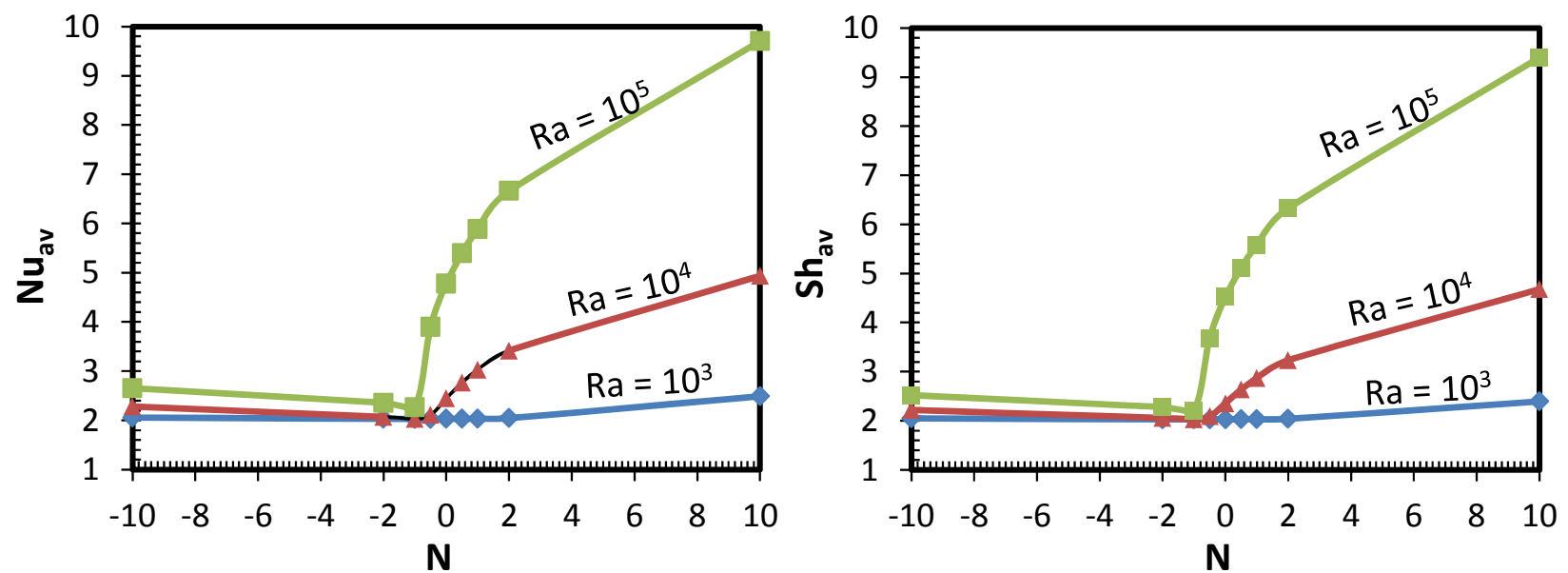

Fig. 10 Average Nusselt and Sherwood numbers

Figs. 6 and 7 present, respectively, the iso-surfaces of temperature and the iso-surfaces of concentration for different buoyancy ratios. First of all, it is noted that the maps of temperature and concentrations are similar to all the studied cases due to the chosen Lewis number which is neat to unity. For $\mathrm{N}<-1$, the isotherms and iso-concentrations present horizontal stratifications in the lower part of the cavity and excessive gradients near the lower part of the inclined cold wall and they become bent on approaching the sloping top wall. For $\mathrm{N} \geq-1$, they become increasingly distorted in the core region and horizontal stratification persists only in the right corner of the lower part of the cavity. These distortions are more notable for highest buoyancy ratios. This result is more tangible from Fig. 8, representing the isotherms and isoconcentrations in the central XY-plan.

The local Nusselt and Sherwood numbers at the hot bottom wall are illustrated in Fig. 9. As seen from the figure, Nusselt and Sherwood numbers values decrease from left to right (away from the adiabatic vertical wall) but they are distributed almost in a symmetric manner regardless of buoyancy ratio. However, increasing this parameter is accompanied by an increase of both Numbers which means an enhancement of heat and mass transfer. Mean Nusselt and Sherwood numbers (Fig. 10), present the same profile versus the buoyancy ratio, by exhibiting a minimum for $\mathrm{N}=-1$. The highest values are obtained for the highest of both Rayleigh number and buoyancy ratio.

Fig. 11 shows the local entropies generations in the central plan. For both thermal and compositional dominated flow cases, the generated entropies are concentrated near of the active walls. For $\mathrm{N}=$ 2 , the creations of thermal, compositional and friction entropies are mainly localized near the bottom of the hot inclined surface. For $\mathrm{N}=-1$, the generated entropies become more and more distributed in the entire cavity especially the friction one due to the equilibrium between thermal and solutal forces. This equilibrium induces the reduction of the boundary layer phenomenon. Then, the increase of buoyancy ratio $(\mathrm{N} \geq$ 0 ) leads to a distribution increasingly localized near walls. In this case, entropy generation becomes dominated by entropy generation due to friction. For higher N, local entropy generations become closer to the active walls. 
(a) $\mathrm{S}_{\text {th }}$
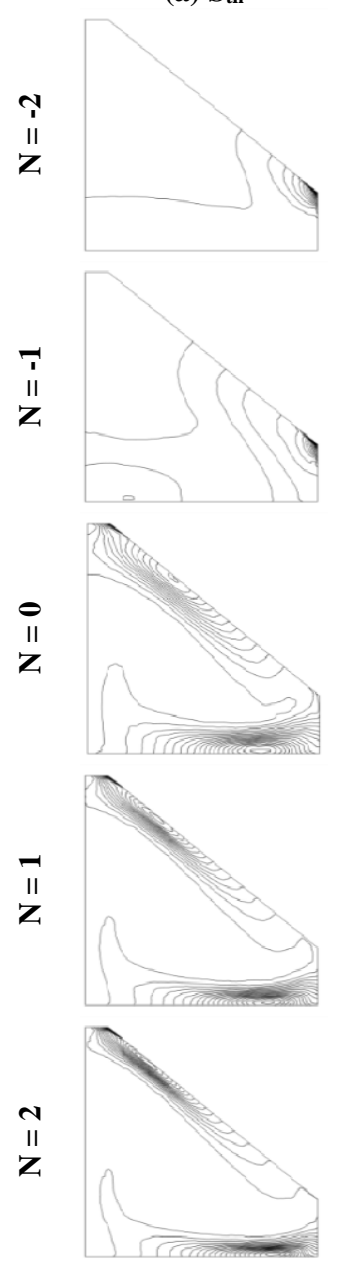

(b) $S_{\text {fr }}$

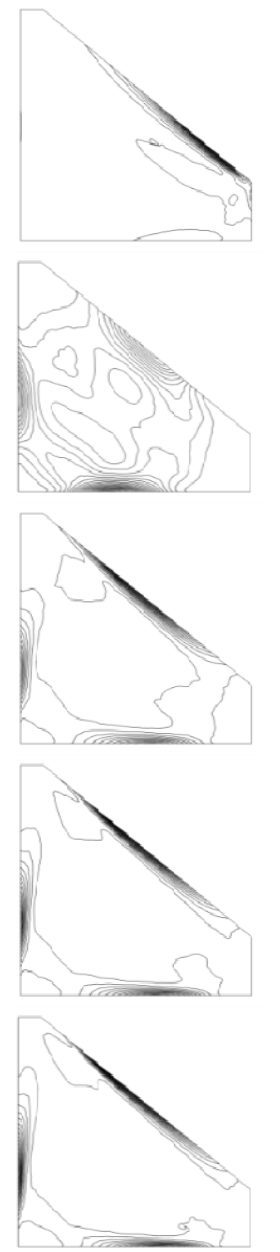

(c) $S_{\text {dif }}$
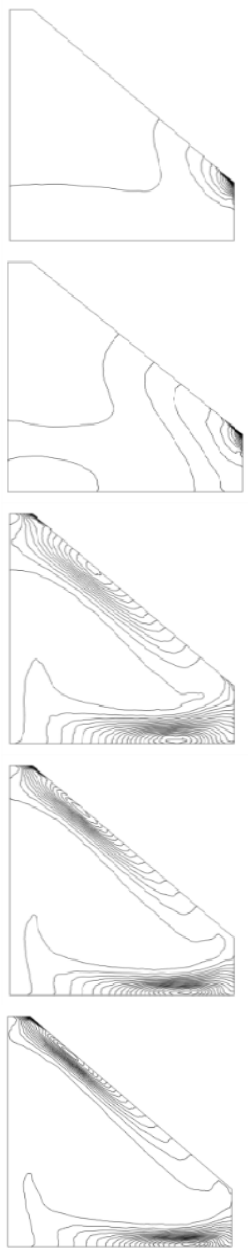

(d) $S_{\text {tot }}$

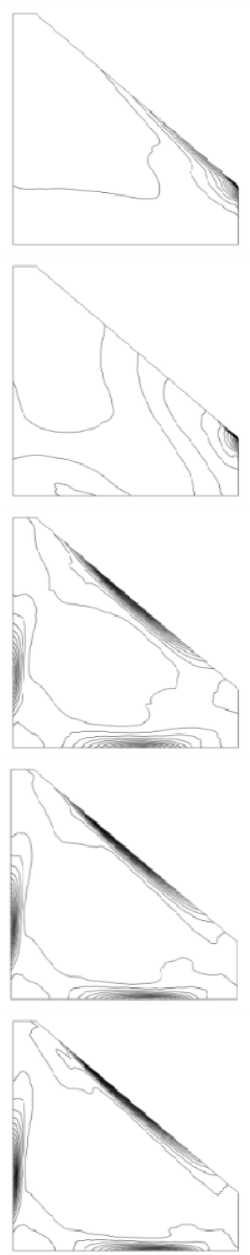

Fig. 11 Local entropies generation in the XY plan for different N; (a) thermal; (b) friction; (c) compositional and (d) total; ; Ra = 10
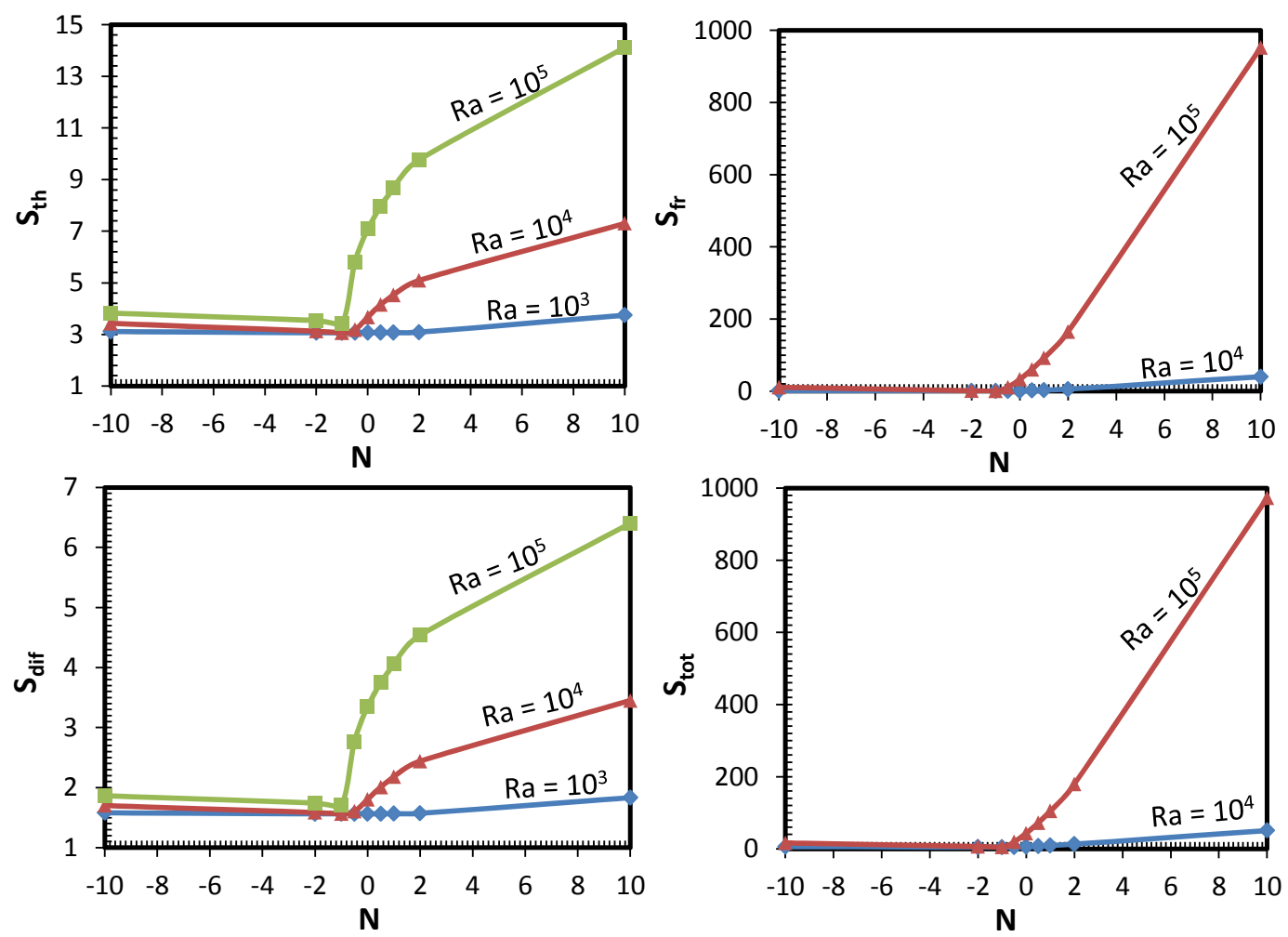

Fig. 12 Entropies generation as function of $\mathrm{N}$ 
Fig. 12 shows that the variation of thermal and compositional entropies are similar versus the buoyancy ratio, and for positive values, they are dominated by friction irreversibility. A minimum in entropy generations occurs for $\mathrm{N}=-1$, due to equilibrium between thermal and compositional forces inducing reduction in velocity, temperature and concentration gradients. The variation of the Bejan number as a function of $\mathrm{N}$ (Fig. 13) shows that only for $\mathrm{N}=1$, irreversibility due to fluid friction is dominated by those due to heat and mass transfers. For $\mathrm{N}=-1$ this variation presents a maximum indicating the net domination of the heat and mass transfer entropy generation.

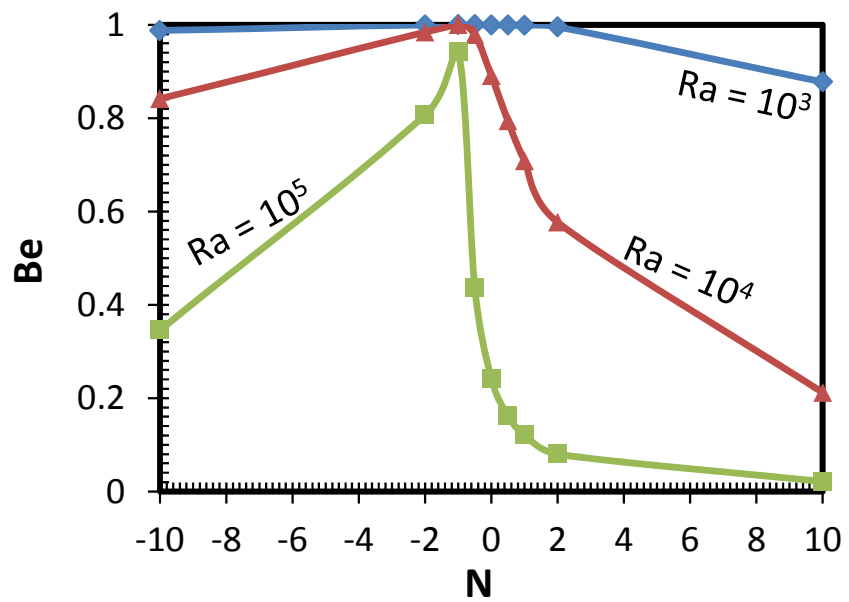

Fig. 13 Bejan number as a function of $\mathrm{N}$

\section{CONCLUSIONS}

A numerical analysis of thermo-solutal natural convection and entropy generation in $3 \mathrm{D}$ trapezoidal solar distiller was performed using computational fluid dynamics (CFD). Some conclusions can be drawn as follows:

- The distributions of thermal and species fields and the structure of the flow are significantly affected by the variation of the buoyancy ratio. Particularly for $\mathrm{N}=-1$, the flow is completely three-dimensional.

- Except for $\mathrm{N}=-1$ where the variation presents a minimum, all types of entropy generation increase by increasing (positively or negatively) the buoyancy ratio.

- For all Rayleigh number values, the highest Bejan number is obtained for $\mathrm{N}=-1$, indicating the domination of heat and mass irreversibilities. Otherwise, friction irreversibilities are largely dominant.

- Mean Nusselt and Sherwood numbers present the same variation as a function of buoyancy ratio and display a minimum for $\mathrm{N}=-1$. The highest values are obtained for the highest Rayleigh number and buoyancy ratio.

- $\quad$ The increased buoyancy ratio $(\mathrm{N} \geq 0)$ leads to a distribution increasingly localized near walls. In this case, entropy generation becomes dominated by irreversibilities due to friction

\section{ACKNOWLEDGEMENTS}

The authors would like to thank the Deanship of the Scientific Research of University of Hail, Saudi Arabia for funding and supporting this research project $\left(\mathrm{N}^{\circ} 160755\right)$.

\section{NOMENCLATURE}

$\begin{array}{ll}\mathrm{Be} & \text { Bejan number } \\ \mathrm{C} & \text { Dimensionless concentration } \\ & \left.\left.\left[=\left(C^{\prime}-C_{l}^{\prime}\right) /\left(C^{\prime}{ }_{h}-C_{l}^{\prime}\right)\right]\right)\right] \\ \mathrm{D} & \text { Mass diffusivity }\left(\mathrm{m}^{2} / \mathrm{s}\right) \\ \mathrm{Gr} & \text { Grashof number } \\ \mathrm{k} & \text { Thermal conductivity }(\mathrm{W} / \mathrm{m} . \mathrm{K})\end{array}$

\begin{tabular}{ll}
$\mathrm{Le}$ & Lewis number \\
$\mathrm{N}$ & Buoyancy ratio \\
$\mathrm{N}_{\mathrm{s}}$ & Local generated entropy \\
$\mathrm{Pr}$ & Prandtl number \\
$\mathrm{R}$ & Gas constant $(\mathrm{J} / \mathrm{mol} . \mathrm{K})$ \\
$\mathrm{Ra}$ & Rayleigh number \\
$\mathrm{Ri}$ & Richardson number \\
$\mathrm{Sc}$ & Schmidt number \\
$\mathrm{Sh}$ & Sherwood number \\
$\mathrm{S}^{\prime}{ }_{\text {gen }}$ & Generated entropy $(\mathrm{J} / \mathrm{K})$ \\
$\mathrm{t}$ & Dimensionless time $\left(=t^{\prime} \cdot \alpha / W^{2}\right)$ \\
$\mathrm{T}$ & Dimensionless temperature $\left[=\left(T^{\prime}-T^{\prime}{ }_{C}\right) /\left(T^{\prime}{ }_{h}-T^{\prime}{ }_{C}\right)\right]$ \\
$T^{\prime}{ }_{c}$ & Cold temperature $(\mathrm{K})$ \\
$T^{\prime}{ }_{h}$ & Hot temperature $(\mathrm{K})$ \\
$\vec{V}$ & Dimensionless velocity vector $\left(=\vec{V}^{\prime} \cdot W / \alpha\right)$ \\
$\mathrm{W}$ & Enclosure width $(\mathrm{m})$ \\
& \\
$G r e e k$ symbols & \\
$\alpha$ & Thermal diffusivity $\left(\mathrm{m}^{2} / \mathrm{s}\right)$ \\
$\beta$ & Coefficient of thermal expansion $\left(\mathrm{K}^{-1}\right)$ \\
$\mu$ & Dynamic viscosity $(\mathrm{kg} / \mathrm{m} . \mathrm{s})$ \\
$v$ & Kinematics viscosity $\left(\mathrm{m}^{2} / \mathrm{s}\right)$ \\
$v_{0}$ & Characteristic speed of fluid $(=\alpha / W)$ \\
$\varphi_{i}$ & Irreversibility coefficient \\
$\varphi^{\prime}$ & Dissipation function \\
$\vec{\omega}$ & Dimensionless vector potential $\left(\vec{\psi}^{\prime} / \alpha\right)$ \\
& Dimensionless vorticity $\left(=\vec{\omega}^{\prime} \cdot \alpha / W^{2}\right)$ \\
\hline &
\end{tabular}

Subscripts

$\begin{array}{ll}\mathrm{x}, \mathrm{y}, \mathrm{z} & \text { Cartesian coordinates } \\ \text { dif } & \text { diffusive } \\ \mathrm{fr} & \text { Friction } \\ \text { th } & \text { Thermal } \\ \text { tot } & \text { Total }\end{array}$

Superscript

Dimensional variable

\section{REFERENCES}

A. Abidi, L. Kolsi, M.N. Borjini, H. Ben Aissia, M.J. Safi, 2008, "Effect of Heat and Mass Transfer through Diffusive Walls on Threedimensional Double-diffusive Natural Convection", Numerical Heat Transfer Part A, 53, 1357-1376. http://dx.doi.org/10.1080/10407780801960241

A. Abidi, L. Kolsi, M.N. Borjini, H. Ben Aissia, 2011, "Effect of Radiative Heat Transfer on Three-dimensional Double Diffusive Natural Convection", Numerical Heat Transfer, Part A, 60, 785-809. http://dx.doi.org/10.1080/10407782.2011.627797

A. Bouchekima, B. Gros, R. Ouahes, M. Diboun, 1999, "Theoretical Study and Practical Application of the Capillary Film Solar Distiller", World Renewable Energy Congress, 16, 795-799. https://doi.org/10.1016/S0960-1481(98)00284-5

A. Bouchekima, B. Gros, R. Ouahes, M. Diboun, 2000, "Etude Théorique et Application Pratique du Distillateur Solaire à Film Capillaire", International Journal of Thermal Sciences, 39, 442-459. https://doi.org/10.1016/S1290-0729(00)00216-7 
A. Das, T. Basak, 2016, "Role of Distributed/Discrete Solar Heaters during Natural Convection in the Square and Triangular Cavities: CFD and Heatline Simulations", Solar Energy, 139, 130-153.

https://doi.org/10.1016/j.solener.2016.04.045

A. Khadiri, A. Amahmid, M. Hasnaoui, A. Rtibi, 2010, "Soret Effect on Double-diffusive Convection in a Square Porous Cavity Heated and Salted from Below", Numerical Heat Transfer, Part A, 57, 848-868. http://dx.doi.org/10.1080/10407782.2010.489487

B. Bouchekima, 2003, "A Small Solar Desalination Plant for the Production of Drinking Water in Remote Arid Areas of Southern Algeria", Desalination, 159, 197-204.

https://doi.org/10.1016/S0011-9164(03)90071-3

C. Béghein, F. Haghighat, F. Allard, 1992, "Numerical Study of Double-diffusive Natural Convection in a Square Cavity", International Journal of Heat and Mass Transfer, 35, 833-846.

https://doi.org/10.1016/0017-9310(92)90251-M

D. Gobin, R. Bennacer, 1996, "Cooperating Thermosolutal Convection in Enclosures II. Heat Transfer and Flow Structure", International Journal of Heat and Mass Transfer, 39, 2683-2697.

https://doi.org/10.1016/0017-9310(95)00351-7

H. Ben Niche, S. Bouabdallah, B. Ghernaout and M. Teggar, 2016, "Unsteady Double Diffusive Natural Convection with Dufour and Soret Effects", International journal of heat and technology, 34, 39-46. https://doi.org/10.18280/ijht.340106

H. Han, T.H. Kuehn, 1991, "Double Diffusive Natural Convection in a Vertical Rectangular Enclosure II". Numerical Study, International Journal of Heat and Mass Transfer, 34, 461-471.

https://doi.org/10.1016/0017-9310(91)90265-G

H.K. Wee, R.B. Keey, M.J. Cunningham, 1989, "Heat and Moisture Transfer by Natural Convection in a Rectangular Cavity", International Journal of Heat and Mass Transfer, 32, 1765-1778. https://doi.org/10.1016/0017-9310(89)90058-6

I. Sezai, A.A. Mohamed, 2000, "Double Diffusive Convection in a Cubic Enclosure with Opposing Temperature and Concentration Gradients", Physics of Fluids, 12, 2210-2223.

http://dx.doi.org/10.1063/1.1286422

K. Ghachem, L. Kolsi, C. Mâatki, A. K. Hussein, M. N. Borjini, 2012, "Numerical Simulation of Three-dimensional Double Diffusive Free Convection Flow and Irreversibilities Studies in a Solar Distiller". International Communications in Heat and Mass Transfer, 39, 869-876.

https://doi.org/10.1016/j.icheatmasstransfer.2012.04.010

K. Sampathkumar, T.V. Arjunanb, P. Pitchandia, P. Senthilkumarc, 2010, "Active Solar Distillation, a Detailed Review", Renewable and Sustainable Energy Reviews, 14, 1503-1526.

https://doi.org/10.1016/j.rser.2010.01.023

K.S. Reddy, N. S. Kumar, 2009, "An Improved Model for Natural Convection Heat Loss from Modified Cavity Receiver of Solar Dish Concentrator", Solar Energy, 83, 1884-1892.

https://doi.org/10.1016/j.solener.2009.07.001

L.A. Tofaneli, M.J.S. de Lemos, 2009, "Double-diffusive Turbulent Natural Convection in a Porous Square Cavity with Opposing
Temperature and Concentration Gradients", International Journal of Heat and Mass Transfer, 36, 991-995.

https://doi.org/10.1016/i.icheatmasstransfer.2009.06.018

M. S. Alam, M. M. Rahman, S. Parvin and K. Vajravelu, 2016, "Finite Element Simulation for Heatline Visualization of Natural Convective Flow and Heat Transfer inside a Prismatic Enclosure", International journal of heat and technology, 34, 391-400.

https://doi.org/10.18280/ijht.340307

N. S. Kumar, K.S. Reddy, 2007, "Numerical Investigation of Natural Convection Heat Loss in Modified Cavity Receiver for Fuzzy Focal Solar Dish Concentrator", Solar Energy, 81, 846-855.

https://doi.org/10.1016/j.solener.2006.11.008

R. Bennacer, A.A. Mohamad, D. Akrour, 2001, "Transient Natural Convection in an Enclosure with Horizontal Temperature and Vertical Solutal Gradients", International Journal of Thermal Sciences, 40, 899-910.

https://doi.org/10.1016/S1290-0729(01)01276-5

R. Bennacer, D. Gobin, 1996, "Cooperating Thermosolutal Convection in Enclosures I. Scale Analysis and Mass Transfer", International Journal of Heat and Mass Transfer, 39, 2671-2681. https://doi.org/10.1016/0017-9310(95)00350-9

S. Bouabdallah, B. Ghernaout, M. Teggar, A. Benchatti and F. Z Benarab, 2016, "Onset of Natural Convection and Transition LaminarOscillatory Convection Flow in Rayleigh-Bénard Configuration", International journal of heat and technology, 34, 151-157. https://doi.org/10.18280/ijht.340122

S. Chen, R. Du, 2011, "Entropy Generation of Turbulent Doublediffusive Natural Convection in a Rectangle Cavity", Energy, 36, $1721-1734$

https://doi.org/10.1016/j.energy.2010.12.056

S. Meftah, A. Ibrahim, D. Lemonnier, A. Benbrik, 2009, "Coupled Radiation and Double Diffusive Convection in Nongray air- $\mathrm{CO}_{2}$ and air- $\mathrm{H}_{2} \mathrm{O}$ Mixtures in Cooperating Situations", Numerical Heat Transfer Part A, 56, 1-19.

http://dx.doi.org/10.1080/10407780903107204

S. M. Mirabedin, 2016, "CFD Modeling of Natural Convection in Right-Angled Triangular Enclosures", International journal of heat and technology, 34, 503-506.

http://dx.doi.org/10.18280/ijht.340322

T. Nishimura, M. Wakamatsu, A.M. Morega, 1998, "Oscillatory Double-diffusive Convection in a Rectangular Enclosure with Combined Horizontal Temperature and Concentration Gradients", International Journal of Heat and Mass Transfer, 41, 1601-1611. https://doi.org/10.1016/S0017-9310(97)00271-8

V.A.F. Costa, 2004, "Double-diffusive Natural Convection in Parallelogrammic Enclosures", International Journal of Heat and Mass Transfer, 47, 2913-2926.

https://doi.org/10.1016/j.ijheatmasstransfer.2004.03.008

Y.S. Li, J.M. Zhan, Y.Y. Luo, 2008, "Unsteady Phenomena in the Double-diffusive Convection Flows at High Rayleigh Number", Numerical Heat Transfer, Part A, 54, 1061-1083. http://dx.doi.org/10.1080/10407780802473632 